\title{
A COMPARISION OF LABORATORY DIAGNOSTIC METHODS OF TUBERCULOSIS AND AETIOLOGY OF SUSPECTED CASES OF PULMONARY TUBERCULOSIS
}

\author{
Bhatt CP ${ }^{1,2}$, Timalsina $B,{ }^{2}$ Kutu $B,{ }^{2}$ Pradhan $R,{ }^{2}$ Maharjan $B,{ }^{3}$ Shrestha $B^{3}$ \\ ${ }^{1}$ Kathmandu Medical College Teaching Hospital, Sinamangal Kathmandu Nepal \\ ${ }^{2}$ Nobel College, Sinamangal, Kathmandu Nepal \\ ${ }^{3}$ German Nepal Tuberculosis Project, Kalimati, Kathmandu Nepal
}

\begin{abstract}
Introduction: Tuberculosis remains a worldwide public health problem despite the highly effective drugs and vaccines are available making tuberculosis a preventable and curable disease. The objective of this study was to compare the different laboratory diagnostic methods of tuberculosis and determine its prevalence.
\end{abstract}

Methodology: Morning sputum samples were collected from suspected cases of pulmonary tuberculosis and proceeded for Ziehl Neelsen staining, fluorescent staining (auramin-O) and mycobacterium culture in Lowenstein Jensen medium.

Results: Total 78 suspected cases of pulmonary tuberculosis were included in this study among them 53 were male and 25 were female. Out of 78 cases, 46 cases were found to be culture positive. In culture positive cases $(83 \%)$ were found to be $M$. tuberculosis and $(17 \%)$ were found to be slow grower, fine colonies, AFB positive but niacin test negative (mycobacteria other than M. tuberculosis). In the direct microscopic examination by Ziehl Neelsen stained smear 26 samples were found to be acid fast bacilli and one sample was culture negative but acid fast bacilli positive. In fluorescent stained smear 34 samples were found to be positive for acid fast bacilli and 5 samples were culture negative but acid fast bacilli positive. Culture was accepted as gold standard, the sensitivity of direct microscopic examination was found $56.5 \%$ for Ziehl Neelsen staining and $73.9 \%$ for fluorescent staining respectively.

Conclusion: In culture positive cases M. tuberculosis and mycobacteria other than M. tuberculosis was found to be $83 \%$ and $17 \%$ respectively, it was found higher in male than female. Fluorescent microscopy is superior to Ziehl Neelsen microscopy but gives more false positive result than Z-N staining. Combining of Ziehl Neelsen and fluorescent staining is better than fluorescent staining alone.

Key words: Fluorescent Microscope, Mycobacterium Culture, M. tuberculosis, Ziehl Neelsen Stain.

\section{INTRODUCTION}

Tuberculosis is a disease of poverty affecting mostly young adults in their most productive years. The vast majority of TB deaths are in the developing world. The tuberculosis continues to be a great public health problem in Nepal. The

\begin{tabular}{l}
\hline Correspondence: \\
Dr. Chandra Prakash Bhatt \\
Associate Professor and Head of Department \\
Department of Microbiology, Kathmandu Medical College \\
Teaching Hospital, Sinamangal \\
Kathmandu, Nepal \\
E-mail: drcpbhatt@yahoo.com, drcpbhatt@gmail.com \\
\hline
\end{tabular}

number of new cases is increasing due to failure in early detection and drug resistance. These are the important problem in treatment and cure of the tuberculosis. ${ }^{1}$ Cure of the disease is possible only with correct diagnosis and appropriate treatment. Early and accurate detection of active cases remains an important objective for improved implementation of chemotherapy and for reduction in the spread of the disease. ${ }^{2}$ The diagnosis of tuberculosis is largely based on conventional approaches, which rely on clinical features and the results of $\mathrm{X}$-ray, microscopy and culture examination. ZN staining or fluorescent staining allows highly accurate diagnoses which are widely available, simple and quick method. ${ }^{3}$ The 
diagnosis traditionally depends upon identifying the infective organisms in secretions or tissues of diseased individuals. Sputum is the main sample for pulmonary tuberculosis. ${ }^{4}$

Isolation of organisms is the only definitive currently available mean for the diagnosis of TB. It has specificity that approaches $100 \%$ and also permits susceptibility testing of the isolates. But due to the poor availability of the culturing facilities, the diagnosis is often delayed and majority of the cases are mismanaged by unwise use of antiTB drugs and other antibiotics, which may also result in developing the resistance. Although AFB smears examination (Microscopy) is time honored and economical, but for this technique the yields requirement is between 5000 to 10,000 organisms per ml. ${ }^{5}$ Conventional culture methods are sensitive and can detect 10-100 organisms per sample. However, culture methods are time consuming and take 6-8 weeks for the results. Furthermore, viable organisms are needed for culture. ${ }^{6,7}$

Mycobacterium tuberculosis is the most important causative agent of tuberculosis (TB) while nontuberculous mycobacteria (NTM) may play a key role in etiology of TB-like syndromes. ${ }^{8}$ Treatment of TB patients in most countries is based solely on the results of microscopic smear positivity. As such, all sputum smear positive diagnosed patients are indiscriminately placed on DOTS, the current international TB treatment strategy. The implication is that NTM is inappropriately managed with first-line anti-tuberculous drug worsening the patient's condition and raising the risk of drug resistance. ${ }^{9,10}$ Although it is known that most sputum smear positive patients are truly TB patients. ${ }^{11}$ The continued increase in TB drug resistance raises the question on the impact of this indiscriminate use of TB drugs to treat all diagnosed sputum smear positive patients. In this study, the staining methods (Z-N and fluorescent staining) are compared and evaluated taking mycobacterium culture on Lowenstein- Jensen (LJ) medium as gold standard and determine the aetiology of suspected cases of pulmonary tuberculosis.

\section{METHODOLOGY}

A Prospective study was conducted at German Nepal Tuberculosis project; Kathmandu duration of the study was from July 2010 to December 2010 . In this study 78 suspected cases of pulmonary tuberculosis were included.

\section{Sample collection}

Sputum specimen was collected in clean, sterile, leak-proof, wide-mouth containers. The processing of the samples was carried out in a bio-safety cabinet. The sample collected was evaluated in terms of its acceptability, proper labeling such as full name, age, sex, serial number of the patient, date of collection.

\section{Microscopy}

Morning sputum sample was collected, stained by Ziehl Neelsen staining and auramine-O fluorochrome method and observed under compound binocular microscope and fluorescent binocular microscope respectively.

\section{Culture}

For mycobacterium culture sputum sample were decontaminated and centrifuged by using $4 \%$ $\mathrm{NaOH}$, according to modified Petroff method and inoculated into Lowenstein Jensen medium. Lowenstein-Jensen media were incubated at $37^{\circ} \mathrm{C}$ and left in the slanted position for 7 days to permit even distribution of the inoculum over the entire surface of the medium. The tubes are then placed upright and incubation at $37^{\circ} \mathrm{C}$ for $6-8$ weeks.

\section{Observation of colony morphology}

The colonies of $M$. tuberculosis were rough, dry, $3-4 \mathrm{~mm}$ in diameter, raised, and thick with wrinkled surface and an irregular thin margin. They were non-pigmented (off-white to faint buff), tenacious and not easily emulsified.

\section{Identification of $M$. tuberculosis}

For identification of $M$. tuberculosis rate of growth, colonies characters, AFB staining and niacin test were used as per manufacture instruction.

\section{Niacin drop test}

Niacin drop test was performed according to manufacturer instruction to identify M. tuberculosis and differentiated it from mycobacteria other than M. tuberculosis.

\section{Ethical consideration}

The research objective and methods were explained to the patient and informed consent was 
taken from each participant before collection of sputum specimen.

\section{Data analysis}

Data was analyzed by EPI-Info version 3.3.2, document version 8.08 updated Sept 2005 and presented by chart and diagrams.

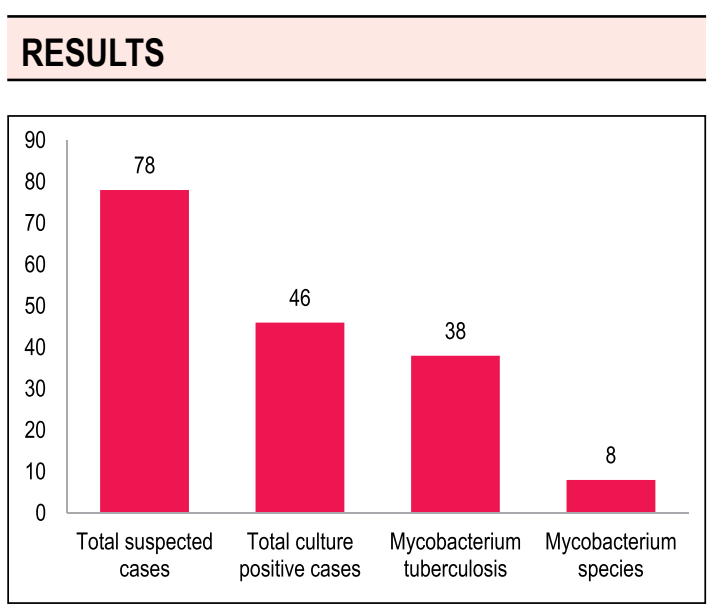

Figure 1. Total suspected and mycobacterium culture positive cases culture positive cases

Out of 46 cultures positive cases, $38(83 \%)$ was found M. tuberculosis and 8 (17\%) was found mycobacteria other than $M$. tuberculosis.

Forty six cases were found to be mycobacterium culture positive. In the direct microscopic

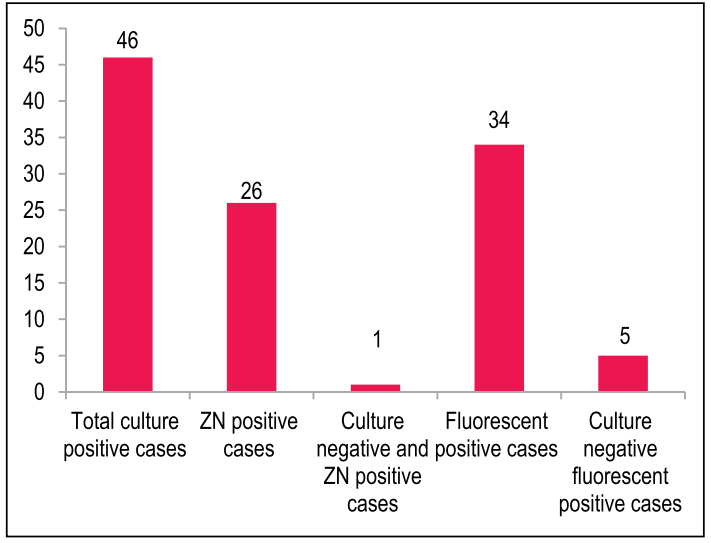

Figure 2. Comparison of mycobacterium culture, Ziehl Neelsen and fluorescent microscopy

examination by Ziehl Neelsen and fluorescent stained smear 26 and 34 samples were found to be acid fast bacilli and one and five samples were culture negative but acid fast bacilli positive respectively.

Mycobacterium species was found higher in male than female and isolated maximum in age group $16-30(43.8 \%)$ followed by the age group $46-60$ $(34.7 \%)$.

Niacin test was performed for 46 culture positive cases. Among them 38 was niacin positive (M. tuberculosis) and 8 was niacin negative (mycobacteria other than M. tuberculosis).

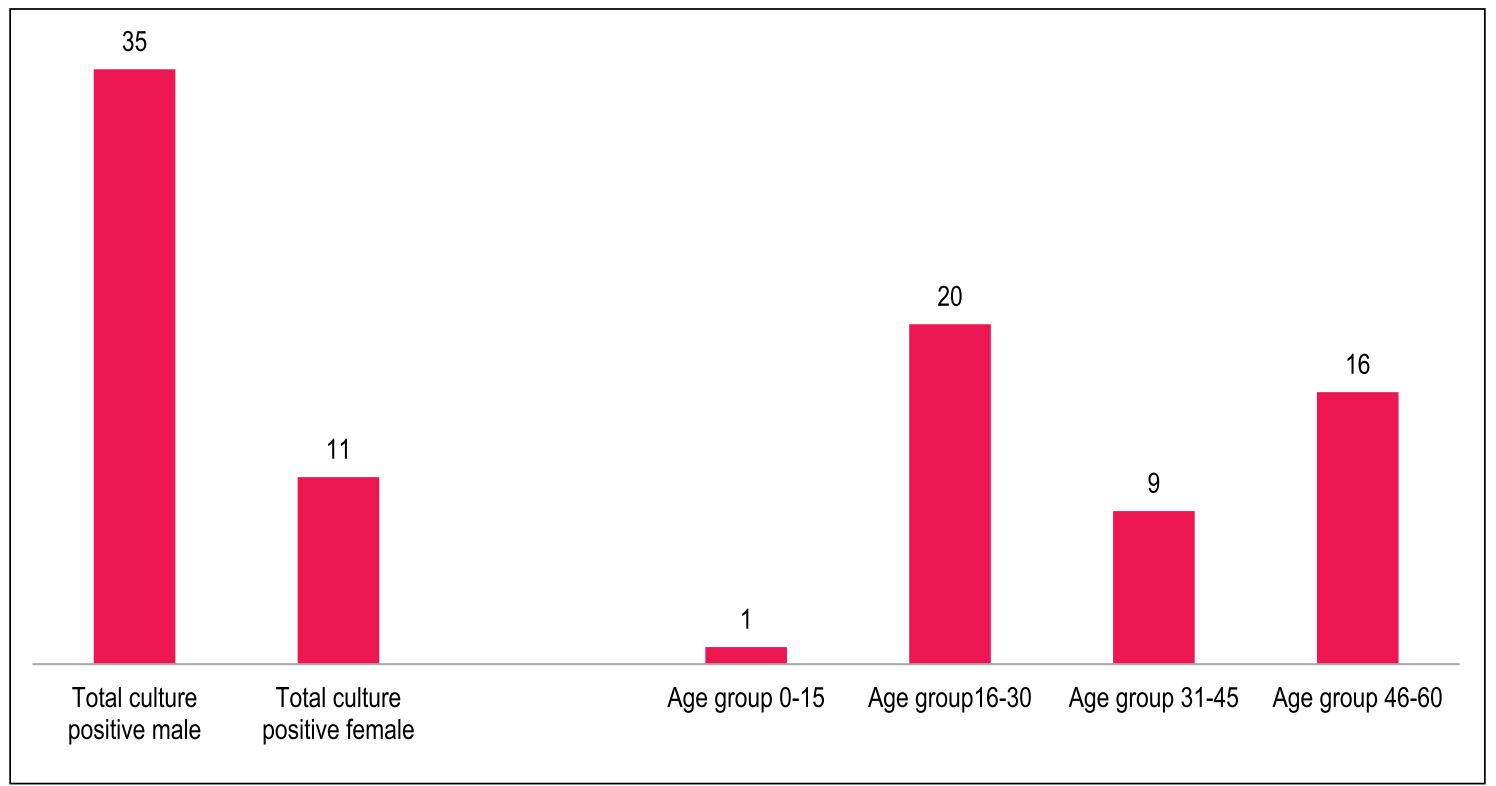

Figure 3. Gender and age wise distribution of positive cases 


\section{DISCUSSION}

According to the WHO guidelines for TB control, patient with more than three weeks history of cough should be screened for pulmonary tuberculosis with direct sputum smear examination for $M$. tuberculosis. Because the clinical signs and symptoms of pulmonary tuberculosis are not specific, the fundamental principal for the diagnosis of tuberculosis is the accurate demonstration of $M$. tuberculosis in a suitable specimen from the suspected cases of pulmonary tuberculosis for the adequate treatment. Either presumptive diagnosis is based on the demonstration of tubercle bacilli in the sputum or M. tuberculosis may be demonstrated by culture. ${ }^{12}$ Detection of smear positive cases is the highest priority in any TB control program, as these cases are infectious and contribute to transmission of disease. Though smear positivity correlates well with infectivity, much of the transmission occurs before the level of bacilli reach $10^{5} / \mathrm{ml}$ in the sputum. ${ }^{13} \mathrm{ZN}$ stain can detect bacilli when they are in the order of $10^{5} / \mathrm{ml}$ of the sputum whereas a more sensitive fluorescent stain can detect in the order of $10^{4} / \mathrm{ml}$ of sputum. ${ }^{14}$

The findings of present study showed that out of 78 cases $46(58.97 \%)$ cases was culture positive. In culture positive cases $83 \%$ was found to be $M$. tuberculosis. In culture positive cases 34 (73.9\%) were positive for fluorescent staining (auramin-0) and $26(56.5 \%)$ were positive for Ziehl Neelsen staining. This finding agrees with the study done in the other parts of the world fluorescent stain is superior to Ziehl Neelsen stain. A study conducted by Laifangbam et al showed that out of 102 patients suspected of pulmonary tuberculosis, $44.1 \%, 71.6 \%$ and $79 \%$ were found positive by $\mathrm{ZN}$, auramine $\mathrm{O}$ staining and culture respectively. ${ }^{13}$ Similar study conducted by Jain et al found sensitivity of ZN was $32.7 \%$ and auramine 0 staining was $41.6 \%{ }^{14}$, Githui et al found sensitivity of $Z N$ was $65 \%$, fluorescent staining was $80 \%{ }^{15}$, Ulukanligil et al found sensitivity of $\mathrm{ZN}$ was $67.6 \%$ and fluorescent staining was $85.7 \%{ }^{16}$ and Prasanthi and Kumar found sensitivity of ZN was $50 \%$ and fluorescent was $69 \% .{ }^{17}$ Fluorchrome stain is more efficient over ZN stain in detecting tubercle bacilli in sputum, especially the paucibacillary cases. Since screening is done under lower power of magnification (400X), fluorescent microscopy has been found to be less time consuming as compared to ZN method (1000X) in the diagnosis of tuberculosis. The fluorescing bacilli are easily identifiable and cause less eye strain. The advantages of sputum smear microscopy is that it has very close relation with infectiousness, patient who are sputum smear positive and culture positive are more likely to be infectious than culture positive but smear_negative. Thus the detection of AFB in sputum smear examination by microscopy play an important part in tuberculosis control programs as transmission of the disease is due mainly to patients whose sputum contains so many organisms that they are detectable by direct microscopy of sputum smear and is helpful to control highly transmission of tuberculosis patients. Ziehl Neelsen staining is rapid and inexpensive but requires a high amount of organisms in the specimens. Fluorescent microscopy is more significant than ZN microscopy but even has chance of observing false negative. Culture along with fluorescent microscopy should be the method of choice for the detection of TB cases in-spite of its time consuming demerit. The detection of TB cases on molecular level is also in practice in our country but due to the lack of molecular expertise, highly expensive equipment need and high test charges these techniques are not so common. Finding of this study suggested that at least fluorescent microscopy and culture must be recommended and should not be rely on clinical symptoms only for the treatment of tuberculosis cases.

Out of 46 culture positive cases 35 were male and 16 were female. In this study $76 \%$ of patients accounted male is higher incidence than the study conducted by Bhatt et al (64\%) $)^{18}$ and Bam (65\%). ${ }^{19}$ This finding agrees with the study conducted in National Tuberculosis Center Thimi, Bhaktapur, Nepal by Bhatt et a ${ }^{20}$ it was found that the incidence of pulmonary tuberculosis was higher in male $75 \%$ than female $25 \%$. In almost all areas where the TB is the public health problem, the incidence of TB among women is less than man. Gender is not merely the biological difference but the differences between men and women in their roles, behaviors, expectations and opportunities within a social cultural and economic context. Nepalese society encounters gender disparities profoundly in many aspects in their lives such as, education, job opportunities, food \& nutrition, morbidity \& mortality pattern of diseases and health care. The low status 
accorded to women in male dominated country like Nepal, their limited decision making power, restricted mobility and poor access to health care resources make them particularly vulnerable to ill health and reduce opportunities in accessing basic and available health care.

The majority $(67.3 \%)$ of patients belongs to economically active young age group 16-45 years. The proportion was nearly same as reported by Bhatt et $\mathrm{al}^{18}(62.7 \%)$ of the respondents belong to 21-50 years age group and Bam ${ }^{19,20}$ reported $(95 \%)$ was $15-54$ years age group. More than $90 \%$ of global TB cases and death occur in the developing world, where $75 \%$ of cases are within the economically most productive age group (1554 years). An adult with TB (in the developing world) loses on average 3-4 months of work time and the economic losses to the family and community are staggering. The estimates suggest a loss of $20-30 \%$ of annual household income and, if the person dies of the disease, an average of 15 years of lost income. ${ }^{21}$ The finding of this study showed that M. tuberculosis was found to be $83 \%$ and mycobacteria other than $M$. tuberculosis was $17 \%$. Similar study conducted by Shanker et al found $7.9 \%$ were atypical mycobacterium. ${ }^{22}$ Kumar and Khurana found incidence of $M$. tuberculosis and atypical mycobacteria $91.6 \%$ and $8.4 \%$ respectively. ${ }^{23}$ Other study conducted by Bhatt et al found all the isolates were M. tuberculosis. ${ }^{18}$ Mycobacteria have been isolated from many sources including soil, animal and human faces, marshland, water (including lakes, rivers, estuaries, swimming pools, aquaria, and domestic water supplies), vegetation and human skin. ${ }^{24}$ Definitive diagnoses of pulmonary nontuberculous mycobacteria infection are difficult. Because the organisms are often saprophytes, they may colonize airways rather than infect them. Cultures can be falsely positive in patients with chronic lung disease and falsely negative in infected patients without cavities. ${ }^{25}$ Thus, identification of acid-fast bacilli at microscopy or isolation of nontuberculous mycobacteria in culture by itself is not enough evidence for establishing the diagnosis. ${ }^{26}$ Further there is cross-reactivity between MAC and $M$. tuberculosis on the purified protein derivative standard test. ${ }^{27}$

Sample size of this study is small and for identification of $M$. tuberculosis rate of growth, colonies characters, AFB staining and biochemical testonly niacin testwas performed. So that itwas not possible to identify niacin negative mycobacterium species (mycobacteria other than M. tuberculosis). It is therefore recommended that a large sample size that would cover rural and urban area of Nepal to compare different diagnostic methods, determine prevalence of $M$. tuberculosis and identification of mycobacterium species different biochemical tests should be considered in further investigation.

\section{CONCLUSIONS}

The findings of this study showed that the efficacy of fluorescence microscopy proved to be much higher than conventional light microscopy. Combining of Ziehl Neelsen and fluorescent staining was better than fluorescent staining (auramine-O) alone both the staining significantly improve sensitivity and percentage of false negative result. In culture positive cases $M$. tuberculosis and mycobacteria other than M. tuberculosis was found to be $83 \%$ and $17 \%$ respectively, it was found higher in male than female. Diagnosis of pulmonary atypical mycobacterium infection may be difficult. The clinical and radiographic manifestations of infection are variable and frequently overlap. For the starting appropriate treatment of atypical mycobacterium diagnosis is the first step that assures full chances of cure because atypical mycobacterium are resistant to standard regime for tuberculosis.

\section{REFERENCES}

1. GC G, Rijal B, Sharma A P. Surveillance of antitubercular drug resistance. Journal of Nepal Medical Association. 2002;41:311-13.

2. Grange M. John. Current topics in infection series 1, Mycobacterial disease, Elsevier North Holland, 1980;1-70.

3. Greenwood D, Slack C.B. Richard, Peutherer F. John. Medical Microbiology, $16^{\text {th }}$ Edition, Elsevier, 2006;200-08.

4. Forbes BA, Sahm DF, Weissfeld AS. Bailey and Scott's Diagnostic Microbiology, $11^{\text {th }}$ edition, Mosby, 2002;538-68.

5. Levy $H$, Feldman C, Sacho CM, Mevlin HV, Hendback JK and Koornhof $\mathrm{H}$. A re-evaluation of sputum microscopy and culture in the pulmonary tuberculosis. Chest. 1989;95:1193-7 
6. Heifets L, Desmond E. Clinical Mycobacteriology (Tuberculosis) Laboratory: Services and Methods. In: Cole ST, Eisenach L, McMurray DN, et al, editors.Tuberculosis and the Tubercle Bacillus. Washington DC: ASM Press, 2005;49-60.

7. Strumpf IJ, Tsang AY, Sayre JW. Re-evaluation of sputum staining for the diagnosis of pulmonary tuberculosis. Am Rev Respir Dis. 1979;4:599-602.

8. Buijtels PCAM, Van Der Sande MAB, De Graaff $\mathrm{CS}$, et al. Nontuberculous mycobacteria, Zambia. Emerging Infectious Diseases. 2009;15:242-49.

9. Koh WJ, Kwon J. Treatment of tuberculosis patients in the private sector in Korea. Tuberculosis and Respiratory Diseases. 2004;56:443-49.

10. Yim JJ, Han SK. Diagnosis and treatment of nontuberculous mycobacterial pulmonary diseases. Journal of Korean Medical Association. 2005;48:563-70.

11. Idigbe EO, Anyiwo CE, Onwujekwe DI. Human pulmonary infections with bovine and typical mycobacteria in Lagos, Nigeria. Journal of Tropical Medicine and Hygiene. 1986;89:143-48.

12. Suitters BT, Brogger SA. Some aspects of laboratory investigations in a mass campaign against tuberculosis. Bull. World Health Orgn. 1967;36:837-45.

13. Laifangbam S, Singh HL, Singh NB, Devi KM, Singh NT. A comparative study of fluorescent microscopy with Ziehl-Neelsen staining and culture for the diagnosis of pulmonary tuberculosis. Kathmandu University Medical Journal. 2009;27:226-30.

14. Jain A, Bhargava A, Agarwal SK. A comparative study of two commonly used staining techniques for acid fast bacilli in clinical specimens. Indian $\mathrm{J}$ Tuberc. 2003;49:161-2.

15. Githui W, Kitui F, Juma ES, Obwana DO, Mwai J, Kwamasga D. A comparative study on the reliability of the fluorescence microscopy and Ziehl-Neelsen method in the diagnosis of pulmonary tuberculosis. East Afr Med J. 1993;70:263-6.

16. Ulukanligil M, Aslan G, Tasci S. A comparative study on the different staining methods and number of specimens for the detection of acid fast bacilli. Mem Inst Oswaldo Cruz. 2000;95:855-8.
17. Prasanthi K, Kumari AR. Efficacy of fluorochrome stain in the diagnosis of pulmonary tuberculosis co-infected with HIV. Indian Journal of Medical Microbiology. 2005;3:179-85.

18. Bhatt $C P$, Bhatt $A B$, Shrestha $B$. Drug resistant cases of tuberculosis in Directly Observed Treatment Short Course. J Nepal Health Res Counc. 2010;16:44-7.

19. Bam TS. Factors affecting patients' compliance with directly observed treatment short course in Kathmandu urban areas, Nepal. Thesis submitted to master of primary health care management faculty of graduate studies, Mahidol University Thailand; Thailand: Mahidol University Thailand; 2003;1-114.

20. Bhatt $\mathrm{CP}$ and Bam DS. The status of pulmonary tuberculosis in National Tuberculosis Center Thimi, Bhaktapur, Nepal. Journal of Nepal Association for Medical Laboratory Sciences. Mar 2001; 42-43.

21. SAARC TB Centre. Gender differences among TB patients in NTPs within SAARC Member Countries. SAARC TB Centre, Kathmandu Nepal, January 2002.

22. Shanker SV, Jain NK, Chandrasekhar S, Singh MM. Prevalence of atypical mycobacteria in sputum of patients undergoing treatment at a tuberculosis clinic. Indian J Chest Dis Allied Sci. 1989;1:9-13.

23. Kumar R, Khurana S. Incidence of Atypical Mycobacterium in Ludhiana. Indian Journal of Medical Microbiology. 1990;139-44.

24. Grange M. John. Current topics in infection series 1, Mycobacterial disease, Elsevier North Holland.1980;1-70.

25. Erasmus JJ, McAdams HP, Farrell MA, Patz EF Jr. Pulmonary nontuberculous mycobacterial infection: radiologic manifestations. Radio Graphics.1999;19: 1487-1505.

26. Ellis SM, Hansell DM. Imaging of non-tuberculous (atypical) mycobacterial pulmonary infection. Clin Radiol. 2000;57:661-69

27. Aksamit TR. Mycobacterium avium complex pulmonary disease in patients with pre-existing lung disease. Clin Chest Med. 2002;23:643-53 